\title{
BETTER LATE THAN NEVER?
}

Zhongbo Chen, ${ }^{1}$ Wei-Jia Zhang, ${ }^{1}$ Radha Ramachandran, ${ }^{2}$ Yusof Rahman, ${ }^{2}$ Lina Nashef'. 'Department of Clinical Neurology, King's College Hospital, London; ${ }^{2}$ Centre for Inherited Metabolic Disorders, Guy's \& St Thomas' Hospital NHS Foundation Trust

\subsection{6/jnnp-2014-309236.69}

We report the case of a 29 year old woman admitted with increasing seizures for assessment of early onset epilepsy of unknown aetiology. She initially presented with convulsions aged 15 days and was treated with phenobarbital. She was subsequently well with normal development until 9 months of age when she started to regress, with onset of epilepsy at 5 years. Despite trials of many antiepileptic drugs and vagus nerve stimulation, she continued to have frequent seizures. She underwent a neurometabolic and genetic screen and was started on a trial of pyridoxine with concurrent medication adjustments. On early follow-up, the family reported a marked improvement in seizure frequency.

The patient was enterally-fed, making nutritional Vitamin B6 deficiency unlikely. The neurometabolic screen excluded alpha-aminoadipic semialdehyde dehydrogenase deficiency but revealed hyperprolinaemia. We propose that the apparent response to pyridoxine treatment is related to the hyperprolinaemia. Although the mechanisms of pyridoxine-dependent seizures are poorly understood, one possible mechanism in hyperprolinaemia may be inactivation of Vitamin B6 by its metabolite pyrroline-5-carboxylate. This case highlights the need for and potential benefit of reassessing patients with early onset epilepsy and learning difficulty, particularly when associated with regression and intractable epilepsy. 